\title{
Giant left anterior descending artery aneurysm
}

\author{
Jelena Vuckovic Karan ${ }^{1,2}$, Aleksandar M. Milosavljevic ${ }^{2}$, Ksenija Babovic Stanic ${ }^{2}$, Lazar Velicki ${ }^{1,2}$ \\ 1Department of Surgery, Medical Faculty, University of Novi Sad, Novi Sad, Serbia \\ ${ }^{2}$ Department of Cardiovascular Surgery, Institute of Cardiovascular Diseases Vojvodina, Sremska Kamenica, Serbia
}

Adv Interv Cardiol 2020; 16, 1 (59): 112-113

DOI: https://doi.org/10.5114/aic.2020.93919

The prevalence of aneurysmal coronary artery disease in patients undergoing coronary angiography varies from $0.2 \%$ to $10 \%$, with such a wide range primarily reflecting the different angiographic criteria used to define coronary artery aneurysm (CAA) [1]. CAA may infrequently grow large enough to be called "giant", defined as $\geq 8 \mathrm{~mm}$ in diameter [2]. Several clinical and treatment questions on this matter remain unanswered together with the current natural history of this condition.

We present a case of a 63-year-old female patient with a history of coronary artery disease (CAD). The patient was admitted to the cardiac surgery clinic for elective coronary artery bypass grafting. Three years prior to admission, the patient sustained anterior wall ST-elevation myocardial infarction that was treated with fibrinolytic therapy in a regional centre. At that time, the patient was not submitted to coronary angiography. Following the last admission due to progressive chest pain, the patient underwent coronarography which demonstrated a sub-occlusive lesion on the left anterior descending (LAD) artery with a huge mid-level LAD aneurysm (Figure $1 \mathrm{~A}$ ). Echocardiography revealed moderately reduced left ventricular ejection fraction of $45 \%$ and otherwise normal findings. The patient was admitted for surgery. The LAD aneurysm was easily identified, measuring $35 \mathrm{~mm}$ in diameter (Figure $1 \mathrm{~B}$ ). Total exclusion of the aneurysm was performed with clipping the artery proximally and distally in relation to the LAD aneurysm. On-pump left internal mammary artery to LAD anastomosis was subsequently performed. The patient was discharged in a good condition on the $7^{\text {th }}$ postoperative day.

The pathogenesis of CAA is not clearly understood, but there is compelling evidence of associations between certain risk factors and CAAs: genetic susceptibility, CAD, certain vasculitis and connective tissue diseases, and lo- cal wall injury following intracoronary manipulation [3]. CAD was most likely the underlying cause of CAA development in our patient.

Most CAAs are incidentally detected during coronary angiography or computed tomography. The majority of cases remain clinically silent, although a variety of clinical scenarios may develop: 1 - effort angina and acute coronary syndrome due to concomitant coronary artery disease, 2 - distal embolization, 3 -compression of local structures in cases of giant CAAs, 4 - aneurysm rupture, 5 - stress-induced ischemia due to microvascular dysfunction [3].

The treatment of CAAs remains speculative in the absence of clinical trials. Medical therapy generally consists of attempts to prevent thromboembolic complications and mainly relies on using antiplatelet medications and/ or anticoagulants. For patients with coexisting obstructive lesions and symptoms or signs of significant ischaemia, percutaneous and/or surgical coronary revascularisation can safely and effectively restore normal myocardial perfusion. Percutaneous coronary intervention of an aneurysmal vessel has been consistently associated with lower procedural success and a higher rate of complications, especially in the setting of acute coronary syndrome. Surgical tactics include aneurysm ligation with distal bypass grafting, isolated coronary artery bypass grafting, aneurysm plication and saphenous vein patch repair of the aneurysm [4]. Surgical revascularization is the preferred approach for addressing giant coronary aneurysms [5]. Lastly, it is important to acknowledge that the treatment for each patient is individual and based on the location of the aneurysm and clinical context.

\section{Acknowlegdments}

This paper was supported by Serbian Ministry of Education, Science and Technological Development [III41007].

\section{Corresponding author:}

Lazar Velicki, Department of Cardiovascular Surgery, Institute of Cardiovascular Diseases Vojvodina, Put doktora Goldmana 4, 21204 Sremska Kamenica, Serbia, phone: +381 21480 5755, fax: +381 21 661099, e-mail: lazar.velicki@mf.uns.ac.rs

Received: 3.08.2019, accepted; 4.09.2019. 

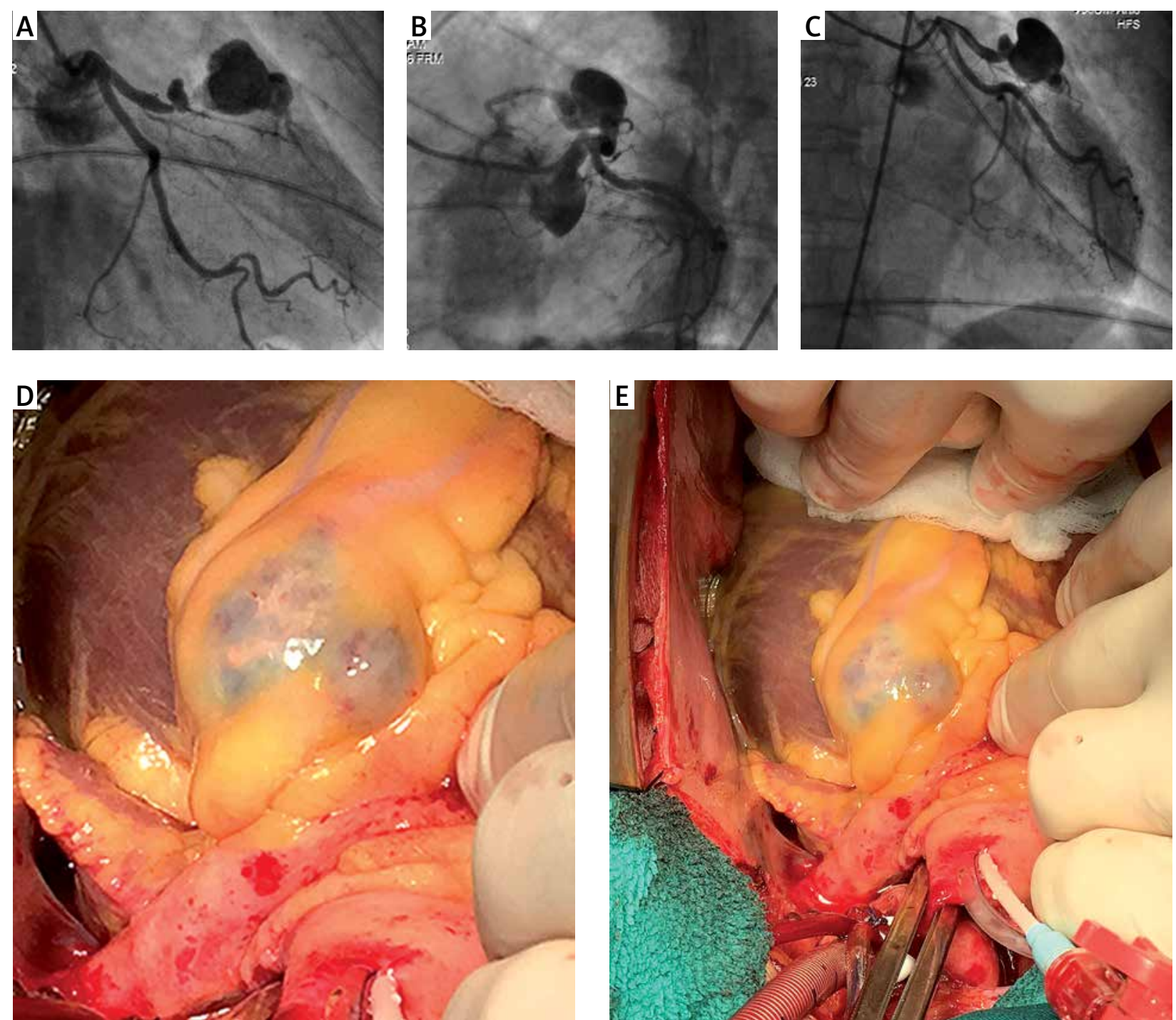

Figure 1. A-C - Coronarography demonstrating large LAD mid-level aneurysm with proximal LAD sub-occlusive lesion. $\mathrm{D}, \mathrm{E}$ - intraoperative finding of giant LAD aneurysm

\section{Conflict of interest}

The authors declare no conflict of interest.

\section{References}

1. ElGuindy MS, ElGuindy AM. Aneurysmal coronary artery disease: an overview. Glob Cardiol Sci Pract 2017; 2017: e201726.

2. Ramos SG, Mata KMA, Martins CCS, et al. Giant right coronary artery aneurysm presenting as a paracardiac mass. Cardiovasc Pathol 2008; 17: 329-33.

3. Kawsara A, Núñez Gil IJ, Alqahtani F, et al. Management of coronary artery aneurysms. JACC Cardiovasc Interv 2018; 11: 1211-23.

4. Crawley PD, Mahlow WJ, Huntsinger DR, et al. Giant coronary artery aneurysms: review and update. Tex Heart Inst I 2014; 41: 603-8.

5. Liu J, Wang X, Sun D, et al. Dual aneurysms of the left anterior descending coronary artery initially detected by echocardiography. Int Heart J 2019; 60: 778-83. 